\title{
A utilização do método canguru em Unidades de Terapia Intensiva Neonatal
}

\author{
The use of the kangaroo method in Neonatal Intensive Care Units \\ El uso del método canguro en las Unidades de Cuidados Intensivos Neonatales
}

Recebido: 08/05/2021 | Revisado: 16/05/2021 | Aceito: 29/05/2021 | Publicado: 01/06/2021

Mariana Pereira Barbosa Silva ORCID: https://orcid.org/0000-0003-0852-8099

Universidade Estadual do Piauí, Brasil

E-mail: marianapbsilvaa@gmail.com

Airton César Leite

ORCID: https://orcid.org/0000-0001-7184-8488

Centro Universitário Santo Agostinho, Brasil

E-mail: ainton.cesar2014@gmail.com

Samuel Lopes dos Santos

ORCID: https://orcid.org/0000-0003-3375-9171

Universidade Federal do Piauí, Brasil

E-mail: samuellopes121314@gmail.com

Maria Vitalina Alves de Sousa

ORCID: https://orcid.org/0000-0003-4448-2489

Centro Universitário INTA, Brasil

E-mail: enf.vitalinaalves@gmail.com

Layanne Cavalcante de Moura

ORCID: https://orcid.org/0000-0003-2781-1076

Centro Universitário UNIFACID, Brasil

E-mail: layannecavalcante@ hotmail.com

Vanessa Maranhão Noleto da Fonseca

ORCID: https://orcid.org/0000-0002-9845-6203

Centro Universitário UNIFACID, Brasil

E-mail: vanessa_maranhao1@ @otmail.com

Simone Barroso de Carvalho

ORCID: https://orcid.org/0000-0001-7428-8420

Universidade Federal do Piauí, Brasil

E-mail: simonebcarvalho2009@hotmail.com

Jocenara Firme de Moura Santos Martins

ORCID: https://orcid.org/0000-0003-4867-0049

Universidade Federal do Piauí, Brasil

E-mail: jocenara_santos@hotmail.com

Alane da Silva Tôrres

ORCID: https://orcid.org/0000-0001-9467-1945

Universidade Federal do Piauí, Brasil

E-mail: alane-torres@hotmail.com

José Ronildo da Costa

ORCID: https://orcid.org/0000-0002-2730-6320 Universidade Regional do Cariri, Brasil

E-mail: ronildo156@hotmail.com

Rosanna Rafena Ribeiro Barbosa

ORCID: https://orcid.org/0000-0003-1777-2245

Associação de Ensino Superior do Piaú, Brasil

E-mail: rrafena@live.com

Janiele Soares de Oliveira

ORCID: https://orcid.org/0000-0003-1468-1452

Associação de Ensino Superior do Piauí, Brasil

E-mail: janysoares276@gmail.com

Rejane Barbosa Ciriaco Pinheiro

ORCID: https://orcid.org/0000-0002-6529-6901

Faculdade de Enfermagem São Vicente de Paula, Brasil

E-mail: rejaneciriaco@ hotmail.com

Amanda Costa Maciel

ORCID: https://orcid.org/0000-0002-2668-037X

Faculdade São Vicente de Pão de Açúcar, Brasil

E-mail: amandacmaciel@ hotmail.com

Gustavo Baroni Araujo

ORCID: https://orcid.org/0000-0002-3162-7477

Universidade Estadual de Londrina, Brasil

E-mail: gustavobaroni13@hotmail.com 


\title{
Nathália Menezes Dias \\ ORCID: https://orcid.org/0000-0002-2166-5293 Universidade Estadual do Pará, Brasil E-mail: menezesdiasnathalia@gmail.com \\ Pâmela Ferreira Brito \\ ORCID: https://orcid.org/0000-0001-6780-4116 Centro Universitário de Belo Horizonte, Brasil \\ E-mail: pamelafbrito@ hotmail.com \\ Joelma Maria dos Santos da Silva Apolinário \\ ORCID: https://orcid.org/0000-0001-9521-9432 \\ Faculdade Maurício de Nassau, Brasil \\ E-mail: jo.silva00@hotmail.com
}

\begin{abstract}
Resumo
A prematuridade é um problema de saúde pública, em que requer a necessidade de internação do recém-nascido (RN) em Unidades de Terapia Intensiva Neonatal (UTIN), no entanto, gera a separação precoce entre mãe e bebê, sendo considerado, pelas mães, um momento devastador e traumático, além de alterar a dinâmica e rotina familiar. Este estudo tem como objetivo discutir a partir de achados na literatura a utilização do método canguru em unidades de terapia intensiva neonatal com enfoque nos seus benefícios. Trata-se de um estudo descritivo, do tipo revisão integrativa de literatura, de caráter qualitativo, a busca dos artigos foi realizada com o auxílio das bases de dados LILACS, SciELO, BDENF, via BVS, e Scholar Google. Os artigos foram coletados no período de abril de 2021. Foram utilizados os descritores: "Método canguru" and "Unidade de terapia intensiva neonatal" and "Recém-Nascido Prematuro", cruzados com o operador booleando "AND", localizados na lista dos Descritores em Ciências da Saúde (DECs). Após a aplicação dos critérios de elegibilidade, restaram 13 estudos que se adequaram ao objetivo proposto pela pesquisa. A utilização do método canguru compreende um novo modelo de cuidado que insere a família no tratamento do neonato, com objetivo de humanizar a assistência. Conclui-se que são amplos os benefícios de tal método, contribuindo significativamente para o desenvolvimento do $\mathrm{RN}$ em vários aspectos, além de promover um maior vínculo entre mãe e bebê.
\end{abstract}

Palavras-chave: Método canguru; Unidade de Terapia Intensiva neonatal; Recém-Nascido prematuro.

\begin{abstract}
Prematurity is a public health problem that requires the need for hospitalization of the newborn (NB) in Neonatal Intensive Care Units (NICU), however, generates a premature separation between mother and baby, being considered by mothers a devastating and traumatic moment, in addition to changing the dynamics and family routine. This study aims to discuss from literature findings the use of the kangaroo method in neonatal intensive care units focusing on its benefits. This is a descriptive study, of the integrative literature review type, of qualitative nature, the search for articles was performed with the aid of the LILACS, SciELO, BDENF, via BVS, and Scholar Google databases. The articles were collected in the period April 2021. The descriptors "Kangaroo Method" and "Neonatal Intensive Care Unit" and "Premature Newborn" were used, crossed with the boolean operator "AND", located in the list of Descriptors in Health Sciences (DECs). After applying the eligibility criteria, 13 studies were left that fit the research objective. The use of the kangaroo method comprises a new model of care that includes the family in the treatment of the newborn, aiming to humanize care. It is concluded that the benefits of this method are broad, contributing significantly to the development of the NB in several aspects, besides promoting a greater bond between mother and baby.
\end{abstract}

Keywords: Kangaroo method; Neonatal Intensive Care Unit; Premature newborn.

\section{Resumen}

La prematuridad es un problema de salud pública que requiere la necesidad de hospitalización del recién nacido (RN) en Unidades de Cuidados Intensivos Neonatales (UCIN), sin embargo, genera la separación precoz entre la madre y el bebé, siendo considerado por las madres, un momento devastador y traumático, además de cambiar la dinámica y la rutina familiar. Este estudio pretende discutir a partir de los hallazgos en la literatura el uso del método canguro en las unidades de cuidados intensivos neonatales centrándose en sus beneficios. Se trata de un estudio descriptivo, tipo revisión bibliográfica integradora, de naturaleza cualitativa, la búsqueda de artículos se realizó con el auxilio de las bases de datos LILACS, SciELO, BDENF, vía BVS, y Scholar Google. Los artículos se recogieron en el periodo de abril de 2021. Se utilizaron los siguientes descriptores: "Método Canguro" y "Unidad de Cuidados Intensivos Neonatales" y "Recién Nacido Prematuro", cruzados con el operador booleano "AND", situado en la lista de Descriptores en Ciencias de la Salud (DECs). Tras la aplicación de los criterios de elegibilidad, quedaron 13 estudios adecuados para el objetivo propuesto por la investigación. La utilización del método kanguru comprende un nuevo modelo de cuidado que inserta a la familia en el tratamiento del neonato, con el objetivo de humanizar la asistencia. Se concluye que los beneficios de este método son amplios, contribuyendo significativamente al desarrollo del NB en varios aspectos, además de promover un mayor vínculo entre la madre y el bebé.

Palabras clave: Método canguro; Unidad de Cuidados Intensivos Neonatales; Recién nacido prematuro. 


\section{Introdução}

A prematuridade é um problema de saúde pública. Anualmente nascem 15 milhões de bebês pré-termos no mundo, uma incidência aproximada de um a cada dez nascimentos (Rocha \& Chow-Castillo, 2020). É considerado prematuro crianças cujo nascimento ocorra na idade gestacional inferior a $37^{\mathrm{a}}$ semana e com peso inferior a 2.500 gramas. Em relação ao peso, o recém-nascido pode ser classificado em baixo peso $(<2500 \mathrm{~g})$, muito baixo peso $(<1500 \mathrm{~g})$ e extremo baixo peso $(<1000 \mathrm{~g})$ (Sousa et al., 2019).

A etiologia do parto prematuro é multifatorial e pode ser causado por diversas situações que levem a paciente a ter contrações uterinas antes do tempo previsto, como, por exemplo, gestação múltipla, uso de drogas, abortos de repetição, hipertensão arterial, sofrimento fetal, gravidez na adolescência, entre outros (Santos \& Sapucaia, 2021).

Diante disso, existe a necessidade de internação em Unidades de Terapia Intensiva Neonatal (UTIN) que contribuem significativamente para o aumento da taxa de sobrevida de prematuros (Silva et al., 2018). A UTIN apresenta ambiente terapêutico apropriado para tratamento do $\mathrm{RN}$ em estado grave e além de tecnologia e equipamentos diversificados, apresenta profissionais altamente capacitados e protocolos específicos para assistência ao RN (Silva et al., 2020). No entanto, gera a separação precoce entre mãe e bebê, sendo considerado, pelas mães, um momento devastador e traumático, além de alterar a dinâmica e rotina familiar (Souza et al., 2019).

Mediante os limites impostos pelo nascimento prematuro e a necessidade de internação do RN na UTIN, acredita-se que o método canguru (MC) pode ser uma ferramenta utilizada para auxiliar no processo de adaptação materna à nova realidade, favorecendo a confiança materna, a criação dos laços de apego e contribuindo para um desenvolvimento psíquico e cognitivo saudável para o bebê (Abreu, Duarte \& Dittz, 2020).

O Método Canguru (MC) foi desenvolvido na década de 1970 pelos neonatologistas Rey e Martinez, médicos do Instituto Medico Infantil (IMI) de Bogotá, Colômbia, que iniciaram uma grande transformação na concepção e na forma de lidar com o recém-nascido pré-termo ou baixo peso (RNPT/RNBP) na tentativa de diminuir a mortalidade e solucionar o problema de superlotação das Unidades de Terapia Intensiva Neonatal (UTIN) com substituição das incubadoras (Santos \& Azevedo Filho, 2016).

No Brasil, o MC foi integrado como parte das diretrizes políticas relacionadas à Atenção à Saúde dos RNPTs e de baixo peso, incluídos no Programa de Humanização do Pré-Natal e Nascimento, instituído pelo Ministério da Saúde, sob Portaria/GM nº 693, de 5 de julho de 2000 que adotava o método como estratégia fundamental na promoção de mudanças centradas na humanização da assistência e no princípio de cidadania da família, atualizada por meio da Portaria GM/MS n ${ }^{\circ}$ 1.683, de 12 de julho de 2007 (Ferreira et al., 2019).

O MC se apresenta como uma abordagem de intervenção complementar para promover o contato direto do neonato com a mãe desde o momento em que ambos apresentam condições clínicas para desenvolvê-lo (Dantas et al., 2018).

$\mathrm{O}$ MC é uma tecnologia de cuidado que propicia uma transição suave para a vida extrauterina e que tem na mãe um papel indispensável nos cuidados e tratamentos do bebê. É direcionado aos bebês cujo peso ao nascer seja igual ou inferior a $2.000 \mathrm{~g}$, não apresente patologias, esteja ganhando peso e seja capaz de coordenar os movimentos de sucção e deglutição (Araujo et al., 2016). Esse método vem contribuindo para reduzir as taxas de mortalidade entre RNPT e RNBP (Defilipo, Chagas, Nogueira, Ananias \& Silva, 2017).

A equipe multiprofissional deve orientar as mães acerca da sua atuação no MC, como coparticipante no cuidado e na recuperação de seu filho (Silva et al., 2020). Acolhendo-a, bem como sua família, dando condições à participação ativa dos pais no cuidado ao filho permitindo, assim, o vínculo afetivo (Sousa et al., 2019).

Este estudo tem como objetivo discutir a partir de achados na literatura a utilização do método canguru em unidades de terapia intensiva neonatal com enfoque nos seus benefícios. 


\section{Metodologia}

Trata-se de um estudo descritivo, do tipo revisão integrativa de literatura, de caráter qualitativo. Segundo Mendes, Silveira, \& Galvão (2008) esse tipo de revisão inclui a análise de pesquisas relevantes que dão suporte para a tomada de decisão e a melhoria da prática clínica, permitindo a síntese do conhecimento de um determinado assunto, além disso, permite a síntese de múltiplos estudos publicados possibilitando conclusões gerais a respeito de uma área de estudo específica.

Foi seguida as seguintes etapas: definição do tema e elaboração da questão de pesquisa; elaboração dos critérios de elegibilidade, inclusão e exclusão dos estudos; definição dos descritores, busca na literatura e coleta de dados; análise crítica dos estudos incluídos e discussão dos resultados; e apresentação da síntese da revisão.

Para direcionar a presente revisão delineou-se como questão norteadora: "O que a literatura aborda sobre a utilização do método canguru em recém-nascidos prematuros na unidade de terapia intensiva neonatal?".

Para a construção deste trabalho, a busca dos artigos foi realizada na Biblioteca Virtual em Saúde (BVS), com o auxílio das bases de dados Literatura Latino-Americana e do Caribe em Ciências da Saúde (LILACS), Scientific Electronic Library Online (SciELO), Base de Dados de Enfermagem (BDENF) e Scholar Google. Os artigos foram coletados no período de abril de 2021. Foram utilizados os descritores: "Método canguru" and "Unidade de terapia intensiva neonatal" and "Recém-Nascido Prematuro", cruzados com o operador booleando "AND”, localizados na lista dos Descritores em Ciências da Saúde (DECs).

Foram selecionados como critérios de inclusão: artigos completos disponíveis na íntegra, nos idiomas português, espanhol e inglês, com recorte temporal de 2016 a 2021. E os critérios de exclusão foram: artigos incompletos, duplicados, debates, resenhas, resumos ou artigos publicados em anais de eventos, indisponíveis na íntegra.

Ressalta-se que, quanto aos aspectos éticos, por se tratar de um estudo de revisão, não foi necessário o encaminhamento e aprovação de Comitê de Ética em Pesquisa. No entanto, foram respeitados os preceitos éticos e a garantia dos direitos autorais das obras utilizadas.

\section{Resultados e Discussão}

A seguir, o fluxograma embasado no Preferred reporting items for systematic reviews and meta-analyses (PRISMA) sintetiza a busca dos artigos que compuseram a amostra final da revisão (Figura 1). 
Figura 1: Fluxograma com o processo de seleção dos artigos que compuseram a síntese final.

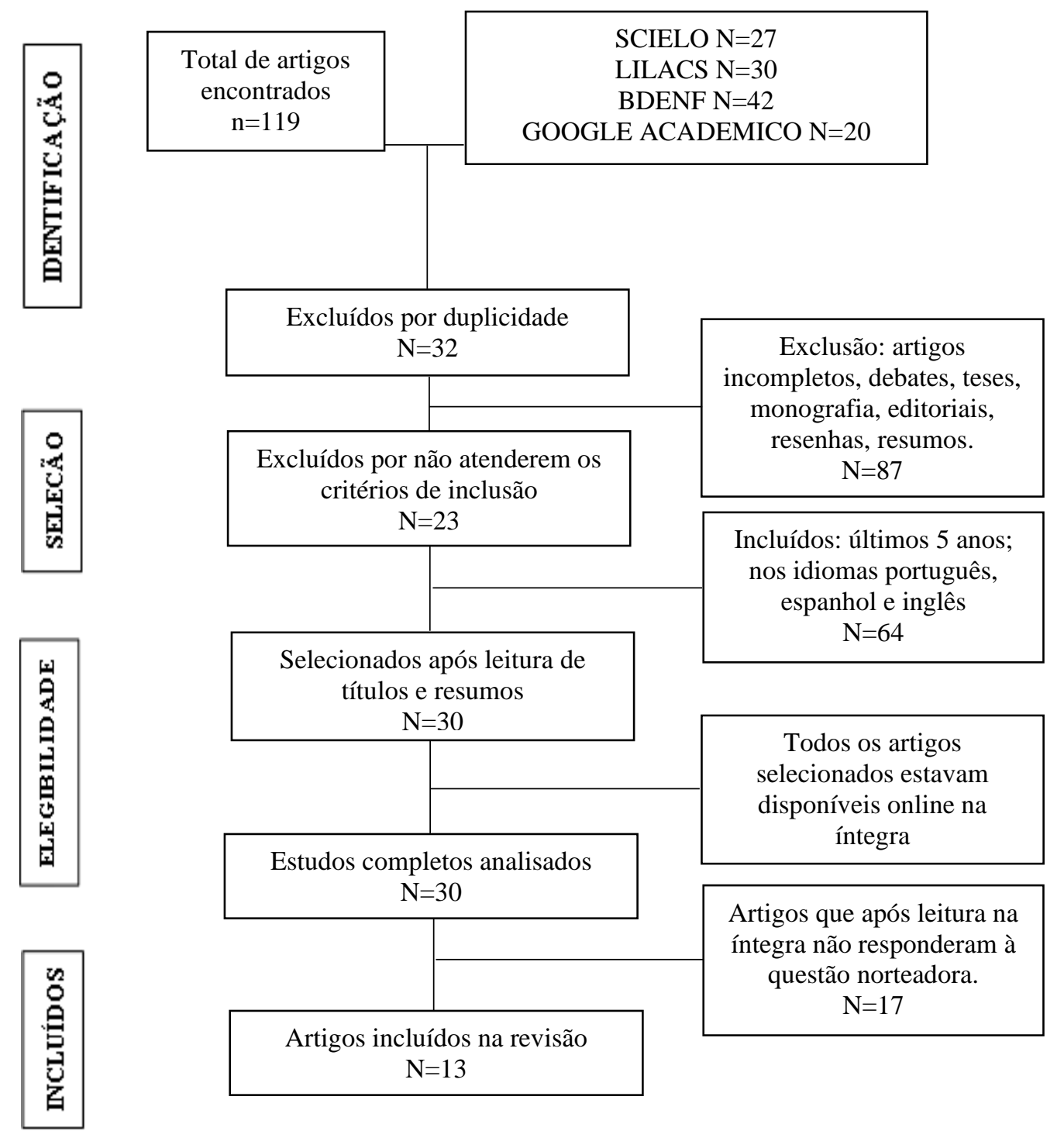

Fonte: Elaborado pelos autores a partir do Fluxograma PRISMA, 2021.

$\mathrm{Na}$ figura 1 observa-se que a partir da coleta de dados, localizaram-se 119 estudos, dos quais 32 foram excluídos por duplicidade e 23 por não atenderem aos critérios de inclusão, restando 64 artigos, que após leitura dos títulos e resumos obteve-se uma amostra de 30 estudos, na primeira etapa da avaliação.

$\mathrm{Na}$ segunda etapa, procedeu-se a leitura dos 30 estudos, desses, 17 foram excluídos por não responder à questão norteadora. Ao final foram incluídos 13 estudos que se adequaram ao objetivo proposto pela pesquisa.

Diante disso, os estudos incluídos para análise final foram organizados em uma planilha de dados contendo as seguintes variáveis: autor, título, ano e metodologia empregada (Quadro 1). 
Quadro 1. Caracterização dos artigos conforme autor, título, ano e metodologia.

\begin{tabular}{|c|c|c|c|c|}
\hline $\mathbf{N}^{o}$ & Autor & Título & Ano & Metodologia \\
\hline 1 & Araujo et al. & $\begin{array}{l}\text { A experiência do método canguru } \\
\text { vivenciada pelas mães em uma } \\
\text { maternidade pública de Maceió/AL }\end{array}$ & 2016 & $\begin{array}{c}\text { Estudo descritivo e exploratório com } \\
\text { abordagem qualitativa }\end{array}$ \\
\hline 2 & Rocha \& Chow-Castillo & $\begin{array}{l}\text { Os benefícios do Método Mãe Canguru na } \\
\text { UTI neonatal }\end{array}$ & 2020 & Revisão da literatura \\
\hline 3 & Ferreira, et al. & $\begin{array}{c}\text { Método canguru: percepções sobre o } \\
\text { conhecimento, potencialidades e barreiras } \\
\text { entre enfermeiras }\end{array}$ & 2019 & $\begin{array}{c}\text { Pesquisa exploratório-descritiva, com } \\
\text { abordagem qualitativa }\end{array}$ \\
\hline 4 & $\begin{array}{l}\text { Santos \& Azevedo } \\
\text { Filho. }\end{array}$ & $\begin{array}{l}\text { Benefícios do método Mãe Canguru em } \\
\text { récem-nascidos pré-termo ou baixo peso: } \\
\text { uma revisão da literatura. }\end{array}$ & 2016 & Revisão da literatura \\
\hline 5 & Sousa, et al. & $\begin{array}{l}\text { Fortalecimento do vínculo entre a família e } \\
\text { o neonato prematuro. }\end{array}$ & 2019 & Estudo qualitativo, descritivo \\
\hline 6 & $\begin{array}{l}\text { Stelmak, Mazza \& } \\
\text { Freire. }\end{array}$ & $\begin{array}{l}\text { O valor atribuído pelos profissionais de } \\
\text { enfermagem aos cuidados preconizados } \\
\text { pelo método canguru. }\end{array}$ & 2017 & $\begin{array}{l}\text { Estudo descritivo, de abordagem } \\
\text { qualitativa }\end{array}$ \\
\hline 7 & Lopes et al. & $\begin{array}{l}\text { Humanização dos cuidados ao recém- } \\
\text { nascido no método canguru: relato de } \\
\text { experiência. }\end{array}$ & 2017 & $\begin{array}{l}\text { Estudo qualitativo, descritivo, do tipo } \\
\text { relato de experiência }\end{array}$ \\
\hline 8 & Dantas et al. & $\begin{array}{l}\text { Percepção das mães sobre a aplicabilidade } \\
\text { do método canguru }\end{array}$ & 2018 & Estudo qualitativo, descritivo \\
\hline 9 & Heck et al. & $\begin{array}{c}\text { Compreensão do sentimento materno na } \\
\text { vivência no método canguru. }\end{array}$ & 2016 & $\begin{array}{c}\text { Estudo descritivo de abordagem } \\
\text { qualitativa }\end{array}$ \\
\hline 10 & Silva, et al. & $\begin{array}{l}\text { Relato de experiência sobre aplicação do } \\
\text { método mãe canguru em uma Unidade de } \\
\text { Terapia Intensiva Neonatal. }\end{array}$ & 2020 & $\begin{array}{c}\text { Estudo descritivo e exploratório com } \\
\text { abordagem qualitativa }\end{array}$ \\
\hline 11 & Zirpoli, et al. & $\begin{array}{l}\text { Benefícios do Método Canguru: Uma } \\
\text { Revisão Integrativa }\end{array}$ & 2019 & Revisão integrativa da literatura \\
\hline 12 & Marques et al. & $\begin{array}{l}\text { Metodologia canguru: benefícios para o } \\
\text { recém-nascido pré-termo. }\end{array}$ & 2016 & $\begin{array}{c}\text { Revisão integrativa, com abordagem } \\
\text { qualitativa, de caráter exploratório, } \\
\text { descritivo e documental }\end{array}$ \\
\hline 13 & Abreu, Duarte \& Dittz. & $\begin{array}{l}\text { Construção do apego entre o binômio mãe } \\
\text { e bebê pré-termo mediado pelo } \\
\text { posicionamento canguru }\end{array}$ & 2020 & $\begin{array}{c}\text { Estudo descritivo e exploratório com } \\
\text { abordagem qualitativa }\end{array}$ \\
\hline
\end{tabular}

Fonte: Dados da Pesquisa (2021).

Nascer prematuramente exige do RN grande esforço para a adaptação extrauterina, devido à imaturidade orgânica e fisiológica, situação que pode causar para o neonato, grandes chances de comprometimentos tanto físicos, quanto mentais (Stelmak, Mazza \& Freire, 2017).

Ao internar o RN na UTIN, dependendo da gravidade em que se encontra, ele precisa ficar dentro de incubadoras sem manipulações, o que acaba prejudicando a criação do vínculo com os pais e os deixando aflitos e tristes por não poderem pegar seu filho no colo. À medida que o RN vai melhorando ou seu estado de saúde não for tão grave, os pais tem que serem incluídos no cuidado do seu filho através de atividades como: troca de fralda; pegar um pouco no colo enquanto a equipe troca os lençóis da incubadora para ajudar no vínculo pais-bebê e para que eles fiquem mais tranquilos, e oferecer o leite materno ordenhado ou, de preferência, no seio, se for possível (Dantas et al., 2018).

O MC compreende um novo modelo de cuidado que insere a família no tratamento do neonato, com objetivo de humanizar a assistência. Esse método recebe essa denominação devido à posição vertical do RN sobre o peito dos pais ou 
familiares, iniciando com o toque e evoluindo progressivamente para a posição canguru, que permite o contato pele a pele, respeitando-se os limites dos pais e do RN (Marques et al., 2016).

O MC permite o contato pele a pele entre a mãe e o bebê prematuro, através de uma faixa de sustentação que envolve o bebê ao corpo da mãe, em posição vertical. Entre as vantagens desse método está o aumento do vínculo mãe e filho devido ao menor tempo de separação entre ambos e a exposição prolongada do bebê à estimulação sensorial, a promoção do aleitamento materno, a manutenção do controle térmico e redução da dor neonatal. Além de permitir um maior estímulo no desenvolvimento neurocomportamental e psicoafetivo do RN e, sobretudo, na redução do período de internação e de risco de infecção neonatal (Rocha \& Chow-Castillo, 2020).

Esse método é utilizado em três etapas, permitindo aos profissionais uma assistência humanizada, em âmbito hospitalar e ambulatorial, à gestante, à puérpera e ao RNPT de baixo peso. A primeira etapa inicia-se no pré-natal da gestação de alto risco, seguido da internação do recém-nascido na UTIN e/ou na Unidade de Cuidado Intermediário Neonatal, onde ocorre o acolhimento da família e a primeira aproximação dos pais e familiares com seus bebês. A segunda etapa inicia-se com o encaminhamento do RN para a Unidade de Cuidados Intermediários Canguru (UCINCa), onde os pais são convidados a estarem juntos aos seus filhos em tempo integral, realizando cuidados diários com orientação da equipe de saúde. Na terceira etapa, o bebê recebe alta para o domicílio, caso atenda aos critérios de estar com o peso mínimo de $1.600 \mathrm{~g}$, esteja apto a sugar o seio materno de forma efetiva e a mãe sentir-se segura e motivada, para a realização dos cuidados domésticos, e que tenha a condição de recorrer à unidade hospitalar em caso de urgência (Ferreira et al., 2019).

Por promover um maior vínculo e envolvimento da família do neonato, esse método aumenta a autoestima, confiança, efetividade e integralidade do cuidado, prolongando o contato pele a pele e desenvolvendo habilidades para a amamentação durante a internação e pós-alta (Sousa et al.,2019).

Conforme o estudo realizado por Abreu, Duarte e Dittz (2020) observa-se que a vivência diária com o bebê pré-termo na UTIN junto à utilização do MC contribui para a mãe desenvolver a autoconfiança e a reestruturação do papel materno além de ser importante para o preparo das mães para o cuidado no domicílio.

O Método canguru foi desenvolvido para estimular as competências do Aleitamento Materno Exclusivo (AME), proporcionando maior frequência, periodicidade e duração com livre demanda da amamentação, única fonte de nutrição e proteção nos primeiros seis meses de vida, uma vez que possui alta concentração de lipídeos totais, nitrogênio, cálcio, vitaminas A, D e E, proteínas com funções imunológicas e ácidos graxos, até a alta hospitalar com acompanhamento adequado pelos profissionais de saúde (Santos \& Azevedo Filho, 2016).

E também pode influenciar, de forma satisfatória, nas variáveis respiratórias, visto que ocorre uma estabilidade da caixa torácica por meio do posicionamento adequado quando colocado em contato pele a pele com a mãe, alterando também as funções hemodinâmicas do recém-nascido (Santos \& Azevedo Filho, 2016).

Segundo o estudo de Zirpoli et al. (2019) observa-se que entre os benefícios da utilização do MC estão a redução da dor e estresse do RN de baixo peso submetido a procedimentos dolorosos na UTIN, diminui o tempo de internação, diminui os custos de recursos humanos e de materiais, aumenta a prevalência do aleitamento exclusivo, aumenta a atividade motora, regula os parâmetros fisiológicos, favorece o sono, revelou-se um aumento significativo da temperatura, da saturação periférica de oxigênio e diminuição significativa da frequência respiratória, facilita a organização comportamental precoce e desenvolvimento do RN, favorece o ganho de peso, e ainda é um instrumento facilitador da amamentação em seio materno.

Devido a UTIN ser um ambiente com excesso de estímulos, o desenvolvimento dos RN pode ser comprometido. A exposição contínua aos mesmos pode causar alteração do padrão de sono, apneia, irritabilidade, crises de choro, podendo refletir no ganho de peso e no desenvolvimento em geral. Além disso, pode ocorrer a liberação do hormônio adrenocorticotrófico, que causa: aumento da adrenalina, elevação da frequência cardíaca, vasodilatação sistêmica, aumento de 
consumo de oxigênio, aumento da pressão sanguínea e intracraniana, predispondo à hemorragia cerebral. Diante disso, o MC busca preservar o RN de ruídos, excessos de luminosidade, excesso de odores, manuseio mínimo, buscando uma aproximação com o ambiente uterino, proporcionando conforto ao $\mathrm{RN}$, minimiza os riscos de iatrogenias e evita prejuízos ao desenvolvimento infantil (Stelmak, Mazza \& Freire, 2017). E ainda pode minimizar a sensação dolorosa do recém-nascido diante das inúmeras intervenções a que é submetido nesse ambiente (Silva et al., 2020).

Os medos, dúvidas e inseguranças quanto à utilização do $\mathrm{MC}$ e ao manejo do recém-nascido prematuro, bem como o estado de agitação, angústia e ansiedade na expectativa da volta para casa, constituem as principais dificuldades encontradas pelas mães, e que refletem diretamente no desenvolvimento do bebê e na eficácia do método (Araujo et al., 2016).

É recomendado que os profissionais responsáveis pela assistência, permaneçam, continuamente, junto ao binômio mãe-bebê, e desenvolvam ações no intuito de minimizar o impacto negativo característico da situação (Abreu, Duarte \& Dittz, 2020).

A equipe deve proporcionar conforto e orientar sobre os procedimentos a serem realizados no RN, incentivando a presença dos familiares, principalmente da mãe, para que estes percebam um ambiente aconchegante e acolhedor, composto por profissionais competentes e habilitados para realizarem os cuidados que o filho necessita (Heck et al., 2016).

A equipe multiprofissional de saúde deve estabelecer uma comunicação efetiva com as mães e familiares durante o período de internação do paciente, visando promover uma maior participação dos mesmos na assistência ao RN, e encorajar os familiares para a execução do MC, que contribui efetivamente para o aumento do vínculo (Lopes et al., 2017).

Ao estabelecer vínculo através do MC, promove-se o reconhecimento e a identificação do jeito de ser entre mãe, pai e filho, tornando-os mais seguros e confortáveis. Nesse sentido, o MC colabora para o enfrentamento das adversidades que podem comprometer o neonato e sua mãe no ambiente hospitalar, promovendo assim uma assistência em saúde integral e humanizada (Lopes et al., 2017).

\section{Conclusão}

Esse estudo é relevante à medida que proporciona conhecer os benefícios da utilização do método canguru na UTIN. Observa-se que são amplos os benefícios de tal método, contribuindo significativamente para o desenvolvimento do RNPT em vários aspectos, além de promover um maior vínculo entre mãe e bebê.

A equipe deve estimular o método canguru apoiando os pais durante todo o processo, sendo empáticos com os familiares. E ainda, ressalta-se a necessidade dos profissionais de saúde se capacitarem para promover esclarecimentos e promover o suporte necessário aos familiares, trabalhando, portanto de forma eficiente e eficaz para prestar uma assistência digna e de qualidade.

Reitera-se a necessidade de novos estudos que avaliem mais benefícios do método canguru na padronização do cuidado aos recém-nascidos prematuros, disseminando assim a utilização da técnica por profissionais nas instituições.

Não houve limitações quanto à elaboração deste estudo, visto a literatura abordar amplamente o tema em questão. No entanto, torna-se importante a realização de novas pesquisas com a finalidade de ampliar a produção científica a respeito dessa temática tão relevante, sendo de fundamental importância, no sentido de aprofundar discussões que possam contribuir para a melhoria do atendimento e prognóstico do RNPT.

\section{Referências}

Abreu, M. Q. S., Duarte, E. D., \& Dittz, E. S. (2020). Construção do apego entre o binômio mãe e bebê pré-termo mediado pelo posicionamento canguru. Revista de Enfermagem do Centro-Oeste Mineiro, 10, e3955.

Araujo, A. M. G., Melo, L. S., Souza, M. E. D. C. A., Freitas, M. M. S. M., Lima, M. G. L., \& Lessa, R. O. (2016). A experiência do método canguru vivenciada pelas mães em uma maternidade pública de Maceió/AL. Rev. iberoam. educ. investi. Enferm., 6(3), 19-29. 
Dantas, J. M., Leite, H. C., Querido, D. L., Esteves, A. P. V. S., Almeida, V. S., Haase, M. M. M.C., \& Labolita, T. H. (2018). Percepção das mães sobre a aplicabilidade do método canguru. Revista de Enfermagem UFPE on line, 12(11), 2944-2951.

Defilipo, E. C., Chagas, P. S. C., Nogueira, C. C. L., Ananias, G. P., \& Silva, A. J. (2017). Kangaroo position: Immediate effects on the physiological variables of preterm and low birth weight newborns. Fisioterapia em Movimento, 30(Suppl. 1), 219-227.

Ferreira, D. O., Silva, M. P. C., Galon, T., Goulart, B. F., Amaral, J. B., \& Contim, D. (2019). Método canguru: percepções sobre o conhecimento, potencialidades e barreiras entre enfermeiras. Escola Anna Nery, 23(4), e20190100.

Heck, G. M. M., Lucca, H. C., Costa, R., Junges, C. F., Santos, S. V., \& Borck, M. (2016). Compreensão do sentimento materno na vivência no método canguru. Revista de Enfermagem da UFSM, 6(1), 71 - 83.

Lopes, T. R. G., Oliveira, S. S., Pereira, I. R. B. O., Romeiro, I. M. M., \& Carvalho, J. B. L. (2017). Humanização dos cuidados ao recém-nascido no método canguru: relato de experiência. Revista de Enfermagem UFPE on line, 11(11), 4492-4497.

Marques, C. R. G., Neris, I. L. F., Carvalho, M. V. A., Menezes, M. O., \& Ferrari, Y. A. C. (2016). Metodologia canguru: benefícios para o recém-nascido pré-termo. Caderno De Graduação - Ciências Biológicas E Da Saúde - UNIT - SERGIPE, 3(3), 65-78.

Mendes, K. D. S., Silveira, R. C. C. P., \& Galvão, C. M. (2008). Revisão integrativa: método de pesquisa para a incorporação de evidências na saúde e na enfermagem. Texto \& Contexto - Enfermagem, 17(4), 758-764.

Rocha, A. M., \& Chow-Castillo, L. A. (2020). Os benefícios do Método Mãe Canguru na UTI neonatal. Educandi \& Civitas, 3(1), 1-16.

Santos, A. P., \& Sapucaia, C. O. (2021). A influência do Método Canguru no tempo de internação do recém-nascido prematuro em unidades hospitalares: uma revisão integrativa. Rev Pesqui Fisioter.,11(1), 252-272.

Santos, M. H., \& Azevedo Filho, F. M. (2016). Benefícios do método Mãe Canguru em récem-nascidos pré-termo ou baixo peso: uma revisão da literatura. Universitas: Ciências da Saúde, 14(1), 67-76.

Silva, A. S., Costa, J. P., Figueiredo, L. S. M., Menezes, J. V., Gandra, V. D., Rodrigues, T. D. N., Silva, F. J. S., \& Paineiras-Domingos, L. L. (2020). A Importância do Método Mãe Canguru na Unidade de Terapia Intensiva Neonatal: uma Revisão de Literatura. Rev Bras Terap e Saúde, 10(2), 1-6.

Silva, J. M. Q., Almeida, M. S., Coelho, E. A. C., Anjos, K. F., Borges, T. P., \& Medeiros, Í. F. (2020). Aprendizados e cuidados de mães no Método Canguru. Rev baiana enferm. 34, e36994.

Silva, P. L. N., Barbosa, S. L., Rocha, R. G., \& Ferreira, T. N. (2018). Experience and needs of parents from premature neonates hospitalized in a neonatal intensive care unit. Rev Enferm UFPI, 7(1), 15-9.

Silva, T. L., Oliveira, A. E. C., Carvalho, J. O., Paiva, E. P., Thofehrn, M. B., \& Borel, M. G. C. (2020). Relato de experiência sobre aplicação do método mãe canguru em uma Unidade de Terapia Intensiva Neonatal. Brazilian Journal of health Review, 3(4), 8767-8774.

Sousa, S. C., Medino, Y. M. S., Benevides, K. G. C. B., Ibiapina, A. S., \& Ataíde, K. M. N. (2019). Fortalecimento do vínculo entre a família e o neonato prematuro. Revista de Enfermagem UFPE on line, 13(2), 298-306.

Souza, J. R, Ribeiro, L. M., Vieira, G. B., Guarda, L. E. D. A., Leon, C. G. R. M. P., \& Schardosim, J. M. (2019). Método canguru na perspectiva dos profissionais de saúde de uma unidade de neonatologia. Enfermagem em Foco, 10(2), 30-35.

Stelmak, A. P., Mazza, V. A., \& Freire, M. H. S. (2017). O valor atribuído pelos profissionais de enfermagem aos cuidados preconizados pelo método canguru. Revista de Enfermagem UFPE on line, 11(9), 3376-3385.

Zirpoli, D. B., Mendes, R. B., Barreiro, M. S. C., Reis, T. S., \& Menezes, A. F. (2019). Benefícios do Método Canguru: Uma Revisão Integrativa . Rev Fund Care Online, 11(n. esp), 547-554. 